\title{
Spherical Dirac GJMS operator determinants
}

\author{
J.S.Dowker ${ }^{1}$ \\ Theory Group, \\ School of Physics and Astronomy, \\ The University of Manchester, \\ Manchester, England
}

\begin{abstract}
Motivated by AdS/CFT, the extension is made to spin-half of a scalar calculation of the conformal anomalies and functional determinants of GJMS operators on spheres. The formal aspects are heuristic but sufficient. A Barnes zeta-function representation again proves effective. The determinants are calculated for the two factorisations of the general $\Gamma$-function (intertwiner) form of the GJMS operator, and shown to be equal, even including any multiplicative anomaly. A comment is made on the general eigenvalue problem and a few numerical results are presented.

An alternative approach is detailed for odd dimensions and it is shown that the scalar determinants are expressed in terms of the spinor ones, and vice versa. An explicit, general form is given.
\end{abstract}

\footnotetext{
1dowker@man.ac.uk; dowkeruk@yahoo.co.uk
} 


\section{Introduction}

The Dirac eigenproblem on spheres is a standard spectral topic. In particular cases, cosmologically motivated, it dates back to the 1930s. It has occurred in quantum field theory notions such as the conformal anomaly, effective action and the Casimir effect in Kaluza-Klein geometries but, more lately, in connection with the AdS/CFT correspondence, where spheres can be both boundary and dual bulk. It is the latter topic that provides my motivation for the calculation that follows. Some of the results are known, in one form or another, but I hope that the procedures here will be viewed as relatively rapid, and sometimes more explicit than existing ones.

The idea is to extend the GJMS operator (on spheres) to spinors and to evaluate various spectral invariants which have field theory significance. Although I do not discuss physics here, motivation for the analysis can be found in aspects of the AdS/CFT correspondance in a particular version of which the propagating operators take on a product form similar to the GJMS operator, [1]. I also refer to the papers [2-4] for a more physics oriented perspective and I give some relevant remarks in section 10 .

It is anticipated that the calculation will be very like the ordinary (scalar) case and the reason is brought out in the next section which outlines some very basic spin-half spectral facts. I pass on to define a calculational Dirac GJMS operator and then to evaluate its conformal anomaly and functional determinant. The arising multiplicative anomalies are found. An explicit formula is obtained for odd dimensions and a holographic interpretation made. I then present some numerics based on the derived formulae. Finally, for odd dimensions, an alternative approach, based on a Bessel Laplace transform, is given. It yields the GJMS logdet as a quadrature. The calculation throws up an already computed integral and gives the result as the traditional combination of Dirichlet $\eta$-functions and $\log 2$, which agrees with existing expressions.

\section{Dirac spectrum on spheres}

I set up the situation by recalling the essentials of the Dirac spectrum of $\not \nabla$ on $S^{d}$. This is usually expressed in the form of eigenlevels,

$$
\mu_{n}(a)= \pm(n+a), \quad n=0,1,2, \ldots, \infty, \quad a=\frac{d}{2},
$$


and degeneracies,

$$
g_{d}(n)=2^{[d / 2]}\left(\begin{array}{c}
n+d-1 \\
n
\end{array}\right) .
$$

I have introduced the parameter $a$ because it is instructive to compare these quantities, not with those for a scalar on a full sphere but rather on a hemi-sphere.

I am interested in the (positive) iterated, or squared, Dirac operator, $\nabla^{2}$, with eigenlevels $\lambda_{n}(a)=\mu_{n}^{2}(a)$ and degeneracies, $2 g_{d}(n)$. For comparison the eigenlevels of the scalar operator, $B^{2} \equiv-\Delta_{2}+(d-1)^{2} / 4$, are $\lambda_{n}((d+1) / 2)$ for Dirichlet (D) conditions on the hemisphere rim and $\lambda_{n}((d-1) / 2)$ for Neumann $(\mathrm{N})$. The degeneracies are exactly as in (2), except for the spin factors.

It can thus be seen that the Dirac case is halfway between the scalar D and $\mathrm{N}$ cases. Both types of mixed boundary conditions for the Dirac field on the hemisphere yield the same set of modes. For Dirac, adding the two hemisphere values amounts to just a factor of two. The two boundary conditions might be termed 'self-dual'.

For many purposes it was advantageous in the scalar case not to work with the eigenlevels and their binomial degeneracies but to write the eigenvalues in the way they emerge from separation of variables in conjunction with the recursive, nested structure of the spherical geometry. This yields the non-degenerate form, ${ }^{2}$

$$
\lambda(\mathbf{m}, a)=(a+\mathbf{m} \cdot \boldsymbol{\omega})^{2}, \quad \boldsymbol{\omega}=(1,1, \ldots, 1),
$$

so that the degeneracies of the eigenlevels $\lambda_{n}$ arise through coincidences, i.e. $g_{n}=$ $\left\{\sharp m_{i}: \sum_{i} m_{i}=n\right\}$ which gives the binomial coefficient. The same applies to the Dirac case, which is encompassed in (3) if $a=d / 2$, apart from the spin factors.

The technical object used in the computation of the functional determinant is the spectral $\zeta$-function of the propagation operator. For example, for $\nabla^{2}$ on the full sphere, using (3), it is a Barnes $\zeta$-function,

$$
\zeta_{S^{d}}(s)=\mathcal{S} \zeta_{d}(2 s, a \mid \mathbf{1}), \quad a=d / 2
$$

putting in the spin factors, $\mathcal{S}=2^{[d / 2]+1}$, by hand. ${ }^{3}$

All this is quite standard, apart from the representation (4), and I now turn to the AdS/CFT aspect. I deal primarily with the boundary side.

\footnotetext{
2 One might refer to this as a harmonic oscillator representation.

3 They could be formally included by sums over spin indices in the definition of the $\zeta$-function.
} 


\section{Boundary facts and Dirac GJMS operator}

Considering the round $\mathrm{S}^{d}$ as a conformal boundary, the spectrum of the spinhalf two-point function can be written in terms of the usual Dirac $\mathrm{S}^{d}$ quantities, (1) and (2) as,

$$
\left|\Lambda_{n}\right|=\frac{\Gamma\left(\left|\mu_{n}(a)\right|-k+1 / 2\right)}{\Gamma\left(\left|\mu_{n}(a)\right|+k+1 / 2\right)},
$$

for the eigenlevels, with degeneracy effectively $2 g_{d}(n)$. As before, $a=d / 2$ and $k$ can be considered to be a real variable subject to $0 \leq k<(d+1) / 2$.

This has been given by Allais, [5], used by Klebanov, Pufu and Safdi, [6] and quoted in Aros and Diaz, [2]. Perhaps is not so surprising in view of the fact that the spin-half two-point function differs from the spin-zero one by a gamma matrix factor, and the eigenvalues vary just in the value of the parameter $a$.

This result suggests that one introduces, pragmatically, a Dirac GJMS operator (on a sphere) by,

$$
D_{2 k}(d) \equiv \frac{\Gamma(B+k+1 / 2)}{\Gamma(B-k+1 / 2)},
$$

where $B$ is the pseudo-operator,

$$
B=\left(\nabla^{2}\right)^{1 / 2}=|\not| \mid
$$

expressed formally. The positive/negative modes of $\not \nabla$ will be positive/positive modes of $|\not| \mid$ for any mode expansion in spinor hyperspherical harmonics, $[7,8]$.

An appropriate choice of $k$ for the spinor case is a half integer, $k=l+1 / 2$, because $D_{2 k}$ takes a product form (odd in $B$ ),

$$
\begin{aligned}
D_{2 l+1}(d) \equiv C_{l} & \equiv B \prod_{h=1}^{l}\left(B^{2}-h^{2}\right) \\
& =\prod_{h=-l}^{l}(B+h), \\
& =B^{[2 l+2]}
\end{aligned}
$$

in terms of an (even) central factorial. Then $D_{1}=C_{0}=B$ will give the usual (massless) Dirac numbers. (An empty product is defined as equalling unity.)

In the scalar case, $C_{l}$ is a well known boundary Dirichlet-Robin (pseudo)operator. 


\section{Zeta function and conformal anomaly}

Corresponding to (3), the eigenvalues of $C_{l}$ have the form,

$$
\Lambda_{l}(\mathbf{m}, a)=(a+\mathbf{m} \cdot \boldsymbol{\omega}) \prod_{h=1}^{l}\left((a+\mathbf{m} \cdot \boldsymbol{\omega})^{2}-\alpha_{h}^{2}\right), \quad \alpha_{h}=h,
$$

degenerate only up to spin factors. Apart from the specific value of $a$, these are very similar to the scalar eigenvalues on a $\mathrm{D}$ or $\mathrm{N}$ hemisphere, $[9,10]$, . Therefore, in order to calculate the conformal anomaly and functional determinant, exactly the same route as in [11] can be followed, only the value of $a$ and the meaning of $\alpha_{h}$ have to be changed with an allowance made for the fact that one factor is linear.

One might hope that any interpolation between the integers that arises in the course of the calculation, would agree with the result of a computation from $D_{2 k}$, (6), but agreement is expected in odd dimensions where there is no conformal nor multiplicative anomaly, as will be shown.

The relevant $\zeta$-function is very similar to that defined in [11]

$$
\begin{aligned}
Z_{d}(s, a, l) & =\mathcal{S} \sum_{\mathbf{m}=\mathbf{0}}^{\infty} \frac{1}{\left(\Lambda_{l}(\mathbf{m}, a)\right)^{s}} \\
& =\mathcal{S} \sum_{\mathbf{m}=\mathbf{0}}^{\infty} \frac{1}{(a+\mathbf{m} \cdot \boldsymbol{\omega})^{s}} \prod_{h=1}^{l} \frac{1}{\left.(a+\mathbf{m} \cdot \boldsymbol{\omega})^{2}-\alpha_{h}^{2}\right)^{s}}
\end{aligned}
$$

and the various evaluations go through more or less verbatim. For example the value at $s=0$ can be read off as the average,

$$
\begin{aligned}
Z_{d}(0, a, l) & =\mathcal{S} \frac{1}{(2 l+1)}\left(\zeta_{d}(0, a)+\sum_{h=1}^{l}\left(\zeta_{d}\left(0, a+\alpha_{h}\right)+\zeta_{d}\left(0, a-\alpha_{h}\right)\right)\right) \\
& =\mathcal{S} \frac{(-1)^{d}}{(2 l+1) d !}\left(B_{d}^{(d)}(d / 2)+\sum_{h=1}^{l}\left(B_{d}^{(d)}(d / 2+h)+B_{d}^{(d)}(d / 2-h)\right)\right) \\
& =\mathcal{S} \frac{\left(1+(-1)^{d}\right)}{2(2 l+1) d !}\left(B_{d}^{(d)}(d / 2)+2 \sum_{h=1}^{l} B_{d}^{(d)}(d / 2+h)\right),
\end{aligned}
$$

where I have inserted the appropriate Dirac value, $a=d / 2$ and used a symmetry property of the Bernoulli polynomial. This quantity, which I will refer to as (minus) the conformal anomaly, is thus zero in odd dimensions, as expected. Calculation of the Bernoulli numbers quickly yields the standard Dirac conformal anomaly when $l=0$ as a check. 
As in the scalar case, in this approach there is no need to derive and then expand the degeneracies, which is the traditional method. See Cappelli and d'Appollonio, [12], and Copeland and Toms, [13], for the ordinary Dirac case.

In Fig.1 I plot the spin-half 'conformal anomaly', $c_{d}(l) \equiv-Z(0, d / 2, l)$, against $l$ for a few low dimensions. It computes to a $d$ th degree polynomial in $l$ and I have extended the plot accordingly. The existence of negative modes does not affect this calculation. The polynomials are even in the variable $k=l+1 / 2$ and I list some below.

Fig.1. Conformal anomaly

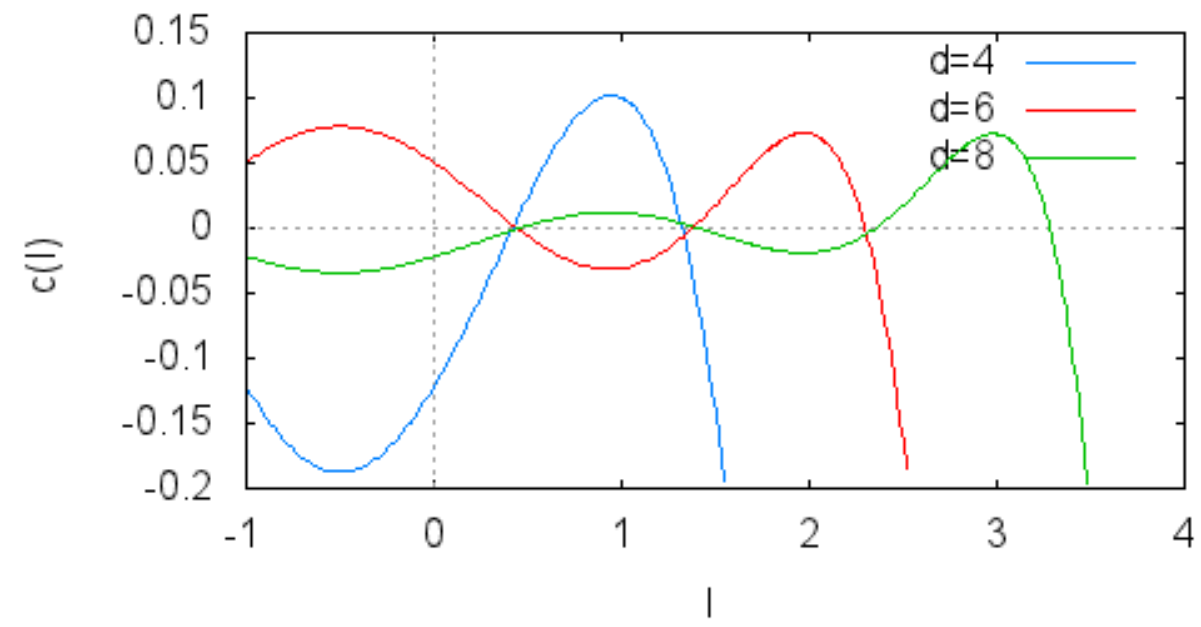

$$
\begin{aligned}
& c_{2}(k-1 / 2)=-\frac{1}{6}\left(4 k^{2}-3\right) \\
& c_{4}(k-1 / 2)=-\frac{1}{720}\left(48 k^{4}-200 k^{2}+135\right) \\
& c_{6}(k-1 / 2)=-\frac{1}{60480}\left(192 k^{6}-2352 k^{4}+7252 k^{2}-4725\right) \\
& c_{8}(k-1 / 2)=-\frac{1}{14515200}\left(1280 k^{8}-34560 k^{6}+284256 k^{4}-774960 k^{2}+496125\right) .
\end{aligned}
$$

I note that the curves intersect approximately at their roots, a fact I cannot interpret.

As in [11] the conformal anomaly can be written more explicitly as an integral on using specific expressions for the Bernoulli polynomials in (11). I find,

$$
Z_{d}(0, a, l)+\delta_{k,(d+1) / 2}=\mathcal{S} \frac{2}{k d !} \int_{0}^{k} d t \prod_{i=0}^{d / 2-1}\left(t^{2}-(i+1 / 2)^{2}\right),
$$


where $k=l+1 / 2$. A holographic discussion is given by Aros and Diaz, [2].

For comparison, the spin-zero expression is,

$$
Z_{d}(0, a, l)+\delta_{k, 2 d}=\mathcal{S}_{0} \frac{2}{k d !} \int_{0}^{k} d t \prod_{i=0}^{d / 2-1}\left(t^{2}-i^{2}\right)
$$

where $\mathcal{S}_{0}=1$. In these expressions for the conformal anomaly, account has been taken of the possible existence of zero modes.

\section{Determinants}

For zeta regularised determinants, one requires the derivative of the $\zeta$-function at $s=0$. As mentioned, the calculational process is virtually identical to that in [11] and, therefore, so is the (intermediate) answer which is,

$$
Z_{d}^{\prime}(0, a, l)=\mathcal{S} \log \left(\frac{1}{\rho_{d}^{l+1}} \Gamma_{d}(a) \prod_{h=1}^{l} \Gamma_{d}\left(a+\alpha_{h}\right) \Gamma_{d}\left(a-\alpha_{h}\right)\right)+\mathcal{S} M^{A}(d, a, l)
$$

where $M^{A}(d, a, l)$ is the multiplicative anomaly. The derivatives of the Barnes function have been formally expressed in terms of multiple gamma functions, the definition being

$$
\zeta_{d}^{\prime}(0, a)=\log \frac{\Gamma_{d}(a)}{\rho_{d}}=\log G_{d}(a) .
$$

$\rho_{d}$ is a normalising modulus, [14], and is independent of $a$.

For the moment, I put the multiplicative anomaly to one side and compactify the product in (15) by using the recursion,

$$
\frac{\Gamma_{d}(a)}{\rho_{d}}=\frac{\Gamma_{d+1}(a)}{\Gamma_{d+1}(a+1)}
$$

which has the effect of bringing in the next higher dimension.

Because I gave no details in [11] I take the opportunity here for a brief explanation.

Substituting (17) into (15) yields,

$$
Z_{d}^{\prime}(0, a, l)=\mathcal{S} \log \left(\frac{\Gamma_{d+1}(a)}{\Gamma_{d+1}(a+1)} \prod_{h=1}^{l} \frac{\Gamma_{d+1}(a+h) \Gamma_{d+1}(a-h)}{\Gamma_{d+1}(a+h+1) \Gamma_{d+1}(a-h+1)}\right)+\mathcal{S} M^{A}
$$


and it is clear there will be considerable cancellation. I find the simple expression, with a holographic flavour,

$$
Z_{d}^{\prime}(0, a, l)=\mathcal{S} \log \frac{\Gamma_{d+1}(a-l)}{\Gamma_{d+1}(a+l+1)}+\mathcal{S} M^{A}(d, a, l) .
$$

This holds whatever the value of $a$. In particular, $a=d / 2$ and $l=0$ gives the ordinary Dirac case, (obtainable directly of course). There can be no multiplicative anomaly, reflected in $M^{A}(d, d / 2,0)=0$. Aros and Diaz, [2], give this logdet in the form,

$$
Z_{d}^{\prime}(0, a, 0)=\mathcal{S} \log G_{d}(a), \quad a=d / 2,
$$

which follows using (17). Klebanov, Pufu and Safdi [6] have given the results of calculations of the determinant based on (5), up to a factor, using the dimensional regularisation approach of Diaz and Dorn, [3].

\section{The other factorisation}

In AdS/CFT the problems that one wishes to solve are associated with the $\zeta$-function of the 'exact' eigenvalues, (5), i.e. ,

$$
\Xi_{d}(s, a, l) \equiv \mathcal{S} \sum_{\mathbf{m}=\mathbf{0}}^{\infty} \frac{1}{(\Lambda(l, \mathbf{m}, a))^{s}},
$$

where, in non-degenerate form,

$$
\Lambda(l, \mathbf{m}, a)=\frac{\Gamma(a+\mathbf{m} \cdot \boldsymbol{\omega}+l)}{\Gamma(a+\mathbf{m} \cdot \boldsymbol{\omega}-l+1)} .
$$

Here $l$ is now a real variable subject to $1 \leq 2 l<d+2$. (For the Dirac case, $a=d / 2$ ).

Whenever $\Lambda$ can be written as a product, which happens when $l$ is either integral or half-integral, all calculations can be effected explicitly, as above. The latter case corresponds to the GJMS operator $P_{2 k}$ treated in [11], but applies here also. It gives the alternative product form (now even in $B$ ),

$$
\bar{C}_{k}(d)=\prod_{h=0}^{k-1}\left(B^{2}-\alpha_{h}^{2}\right), \quad \alpha_{h}=h+1 / 2,
$$

instead of (8). The non-degenerate eigenvalues are

$$
\bar{\Lambda}_{k}(\mathbf{m}, a)=\prod_{h=0}^{k-1}\left((a+\mathbf{m} \cdot \boldsymbol{\omega})^{2}-\alpha_{h}^{2}\right)
$$


and the $\zeta$-function is, of course, a special case of (20),

$$
\bar{Z}_{d}(s, a, k)=\mathcal{S} \sum_{\mathbf{m}=\mathbf{0}}^{\infty} \frac{1}{\left(\bar{\Lambda}_{k}(\mathbf{m}, a)\right)^{s}} .
$$

The technical situation is now identical to that in [11] and yields the intermediate formula,

$$
\bar{Z}_{d}^{\prime}(0, a, k)=\mathcal{S} \log \prod_{h=0}^{k-1} \frac{\Gamma_{d+1}(a+h+1 / 2) \Gamma_{d+1}(a-h-1 / 2)}{\Gamma_{d+1}(a+h+3 / 2) \Gamma_{d+1}(a-h+1 / 2)}+\mathcal{S} M^{B}
$$

Simplification, using (17), results in,

$$
\bar{Z}_{d}^{\prime}(0, a, k)=\mathcal{S} \log \frac{\Gamma_{d+1}(a-k+1 / 2)}{\Gamma_{d+1}(a+k+1 / 2)}+\mathcal{S} M^{B}(d, a, k),
$$

and the point now is that the non-polynomial part of this is identical to that in (19) showing that, apart from any multiplicative anomaly, the interpolations of $\Xi_{d}^{\prime}(0, l)$ provided by the two factorisations are the same. I am not certain of the spectral significance of the structure, (22).

\section{The multiplicative anomaly}

I now turn to the multiplicative anomaly correction, $M^{A}(d, a, k)$, a means of determining which, for the product form (22), has been given in [11] equn. (16). Unfortunately an algebraic error means that the expression for $M_{2}$ there is incorrect. The correct version can be found in [15] and will not be shown here. However I give the corresponding formulae for the product (8) obtained from (10). The answer is very similar. The extra single factor in (9) is easily allowed for and I find,

$$
M^{B}(d, a, l)=M_{1}^{B}(d, a, l)+M_{2}^{B}(d, a, l),
$$

where

$$
M_{1}^{B}(d, a, l)=-\sum_{r=1}^{u} \frac{1}{r}\left(\sum_{h=1}^{l} \alpha_{h}^{2 r}\right) H_{l+1 / 2}(r) N_{2 r}(d, a),
$$

and

$$
M_{2}^{B}(d, a, l)=\frac{1}{2 l+1} \sum_{r=1}^{u} \frac{1}{r} \sum_{t=1}^{u-r} \frac{1}{t}\left(\sum_{i<h=0}^{l-1} \alpha_{i}^{2 r} \alpha_{h}^{2 t}\right) N_{2 r+2 t}(d, a) .
$$


The upper limit $u$ equals $d / 2$ for even dimensions, and $(d-1) / 2$ for odd, i.e. $[d / 2]$. $H_{k}$ is related to the harmonic series, $H(r)=\sum_{n=1}^{r} 1 / n$ by,

$$
H_{k}(r)=H(2 r-1)-\frac{1}{2 k} H(r-1),
$$

and $N$ is the residue at the pole of the Barnes $\zeta$-function,

$$
N_{r}(d, a)=\frac{1}{(r-1) !(d-r) !} B_{d-r}^{(d)}(a) .
$$

These formulae hold for any $a$ and any distribution of the $\alpha_{h}$. To repeat, for $M^{A}, \alpha_{h}=h+1 / 2$ while for $M^{B}, \alpha_{h}=h$. For these values, the sums over the $\alpha$ s in (26) and (27) have a combinatorial significance which I will not invoke at this time.

The sums for $M^{A}$ and $M^{B}$ can be performed for a given $d$, and $a=d / 2$ for Dirac, to give polynomials in $k$ and $l$ respectively. Explicit calculation reveals the equality,

$$
M^{A}(d, d / 2, k)=M^{B}(d, d / 2, l),
$$

if $k=l+1 / 2$, even though $k$ and $l$ have to be integers for the intermediate summations to make sense.

Looking back to (19) and (25) confirms that the full effective actions for the two factorisations are also related algebraically by,

$$
Z_{d}^{\prime}(0, a, l)=\bar{Z}_{d}^{\prime}(0, a, k), \quad \text { if } \quad k=l+1 / 2 .
$$

This gives us confidence to extend the results from the integers to the reals to give the quantity $\Xi^{\prime}(0, a, l)$, as a computable quantity, say (19),

$$
\Xi^{\prime}(0, a, l)=Z^{\prime}(0, a, l) .
$$

In odd dimensions, the multiplicative anomaly vanishes by a symmetry property of the Bernoulli polynomials, just like the conformal anomaly.

In even dimensions, for completeness, I exhibit a few of the polynomials, as functions of $k$, with a factor of $k\left(1-4 k^{2}\right)$ removed,

$$
\begin{aligned}
& M^{A}(2,1, k)=\frac{1}{12} \\
& M^{A}(4,2, k)=\frac{1}{2160}\left(14 k^{2}-39\right) \\
& M^{A}(6,3, k)=\frac{1}{7257600}\left(1392 k^{4}-14016 k^{2}+28745\right) \\
& M^{A}(8,4, k)=\frac{1}{1524096000}\left(4656 k^{6}-111096 k^{4}+749695 k^{2}-1355760\right) .
\end{aligned}
$$


A polynomial ambiguity occurs in other schemes for computing $\log \operatorname{det} D_{2 k}$ and $\log \operatorname{det} P_{2 k},[3,4]$. It is tolerably clear that the multiplicative anomalies displayed here and in [15] are unique, within the present scheme. Given that they should be odd in $k$ and zero for $k=1 / 2$ means that what remains is a polynomial of degree $d / 2-1$ in $k^{2}$ and the unknown coefficients can be determined, with checks, from the values of the polynomial at integer, or at half-integer, $k$, which are known with certainty.

The physical significance of any multiplicative anomaly is problematic because of the uncertainties raised by the necessity of renormalisation. ${ }^{4}$ It is also a quantity manufactured on the $\zeta$-function definition of the functional determinant. Different definitions can give different continuations. However if the $\zeta$-function method is used consistently to compare various quantities then the correct, i.e. appropriate, multiplicative anomalies must be employed.

Because, in $\zeta$-function regularisation, the conformal anomaly drives the infinities (and/or log term), there might be a case for endowing the multiplicative anomalies at the roots of $c_{d}(l),(12)$, with some significance. As displayed in Fig.1 these roots appear to be remarkably stable with varying dimension.

\footnotetext{
${ }^{4}$ A similar comment applies in the AdS/CFT context where eigenvalue prefactors are routinely argued away.
} 


\section{Odd dimensions}

Here there is no multiplicative anomaly and the effective action can be given in terms of elementary functions. I follow exactly the procedure of [11].

The expression (19), equivalently (25), is written in terms of Kurokawa's multiple sine function, essentially by definition, ${ }^{5}$

$$
\begin{aligned}
Z_{d}^{\prime}(0, a, l) & =\mathcal{S} \log \sin _{d+1}(a+k+1 / 2) \\
& =\frac{1}{2} \mathcal{S}\left(\log \sin _{d+1}(a+k+1 / 2)-\log \sin _{d+1}(a-k+1 / 2)\right) \\
& =\frac{1}{2} \mathcal{S} \int_{(d+1) / 2-k}^{(d+1) / 2+k} d z \cot _{d+1}(z) \\
& =-\frac{1}{2 d !} \mathcal{S} \int_{(d+1) / 2-k}^{(d+1) / 2+k} d z B_{d}^{(d+1)}(z) \pi \cot (\pi z) \\
& =\frac{\mathcal{S}}{d !} \int_{0}^{k} d z B_{d}^{(d+1)}((d+1) / 2+z) \pi \cot (\pi z) \\
& \equiv \mathcal{S} \int_{0}^{k} d z P_{s}(d, z) \pi \cot (\pi z)
\end{aligned}
$$

where the polynomial, $P_{s}$, is defined by,

$$
P_{s}(d, z)=\frac{1}{d !} z \prod_{i=1}^{(d-1) / 2}\left(z^{2}-i^{2}\right)
$$

I return to this expression later. It can be used for numerical computation but I prefer the representation in terms of the digamma function that works for both odd and even dimensions. I gave some numbers using this for the spin zero case in [15] and section 11 below contains a few Dirac values.

For the three-sphere, (28) agrees with the expression given by Klebanov, Pufu and Safdi, [6], equn.(66) derived using Diaz and Dorn, [3]. Their $\Delta$ equals $3 / 2-k$ here. The intermediate details would appear to be less elegant.

\footnotetext{
5 I refer to [11] for information on this, and other, multiple functions, with original references.
} 


\section{Holographic interpretation}

Purely by algebra we have reached an equation, (25), which has a holographic look in that the right-hand side refers to one higher dimension. To bring this out explicitly, show the dimension of the operator (7) by $B_{d}$ and note that the $\zeta$-function of $B_{d+1}+k$ is, cf (4), $2 \zeta_{d}(s, d / 2+k+1 / 2)$ so that,

$$
\begin{aligned}
\log \frac{\operatorname{det} B_{d+1}+k}{\operatorname{det} B_{d+1}-k} & =\mathcal{S}_{d+1} \log \frac{\Gamma_{d+1}(d / 2+k+1 / 2)}{\Gamma_{d+1}(d / 2-k+1 / 2)} \\
& =-2 Z_{d}^{\prime}(0, d / 2, k)+\mathcal{S}_{d+1} M(d, d / 2, k)
\end{aligned}
$$

using $(25)$ and $\mathcal{S}_{d+1} / \mathcal{S}_{d}=2$. The left-hand side refers to operators on the $(d+1)-$ sphere.

In odd dimensions there results the $(d+1)$ identity,

$$
\log \frac{\operatorname{det} B_{d+1}+k}{\operatorname{det} B_{d+1}-k}=\mathcal{S}_{d+1} \int_{0}^{k} d z P_{s}(d, z) \pi \cot (\pi z),
$$

and, as pointed out in [11] in the scalar case, by differentiating with respect to $k$ it follows that the residues (times $\mathcal{S}_{d+1}$ ) at the poles of the integrand, which are at $(d+1) / 2+n, n=0,1,2 \ldots$, are the degeneracies of the eigenlevels $\mu_{n}(a)=a+n$ of $B_{d+1},(a=(d+1) / 2)$. By a general theorem, this implies that the integrand is proportional to the spin-half Plancherel measure on the space dual to $\mathrm{S}^{d+1}$, i.e. on the hyperbolic $\mathrm{H}^{d+1}$, and this can be checked from the calculated form, (29). In fact this could be taken as a derivation of the Plancherel measure.

\section{Some formal remarks}

In the, more discussed, scalar case, in free field CFT the two point function on the sphere, $\mathrm{S}^{d}$, is given as (e.g. Gubser and Klebanov, [16]),

$$
\langle\mathcal{O}(\xi) \mathcal{O}(\eta)\rangle=\frac{1}{|\xi-\eta|^{d-2 k}}
$$

where $\xi$ and $\eta$ are the unit vectors of two points on the sphere and $|\xi-\eta|^{2}=$ $2(1-\cos \theta)$ in terms of the radial angle, $\theta$, between these points.

The expression (30) can be derived by conformal transformation from flat space where one has the standard Riesz potential,

$$
\left(\Delta_{2}^{-k} f\right)(x)=\frac{1}{\gamma(k)} \int_{\mathbf{R}^{d}}|x-y|^{2 k-d} f(y) d y
$$


with normalisation,

$$
\gamma(k)=2^{2 k} \pi^{d / 2} \frac{\Gamma(k)}{\Gamma(d / 2-k)} .
$$

Now the stereographic conformal transform of $\Delta_{2}^{k}$ is the GJMS operator, $P_{2 k}$, on the sphere as detailed explicitly by Graham, [17]. So, taking the conformal transform of (31) i.e. ,

$$
\left(P_{2 k}^{-1} f\right)(\xi)=\frac{1}{\gamma(k)} \int_{S^{d}}|\xi-\eta|^{2 k-d} f(\eta) d \eta
$$

would solve the eigenproblem immediately, given $P_{2 k}$ as the ratio of $\Gamma$-functions, as in (6), if this were known independently, say by Branson's intertwiner group theory method.

However, if one wishes to proceed stereographically, it is necessary to note that Graham obtains only the (original) product form of $P_{2 k}$, as in (22). In order to produce the, more general,ratio of $\Gamma$-functions, with $B$ related to the YamabePenrose operator, one can apply the Funk-Hecke theorem to the kernel in (32). This was done by Morpurgo, [18], using an existing Gegenbauer expansion, [19] $\S 10.20$ (6) (he actually quotes equn.(12)), to find the operator $\Gamma$-function form of $P_{2 k}$ from (30) by showing that the eigenlevels have the structure (5), for suitable $\mu_{n}$ with the usual sphere degeneracies ( $k$ will be reversed). The same calculation was done by Gubser and Klebanov, [16] and Allais, [5], for spin-half, in the physical CFT context.

\section{A few computations}

I now give some numerical results by the same method as in [15]. Despite the unknown physical status of the multiplicative anomaly, I present values of the derivative of the $\zeta$-function at zero, say from (25), and still call this the effective action, for short.

I repeat the basic equations,

$$
\log \frac{\Gamma_{d}\left(z_{2}\right)}{\Gamma_{d}\left(z_{1}\right)}=\int_{z_{1}}^{z_{2}} d z \psi_{d}(z)
$$

with,

$$
\psi_{d}(z)=\frac{(-1)^{d-1}}{(d-1) !}\left(B_{d-1}^{(d)}(z) \psi(z)+Q_{d}(z)\right)
$$


where the polynomial $Q$ is given by,

$$
Q_{d}(z)=-(-1)^{d-1} \sum_{n=1}^{d-1} \frac{(-1)^{n}}{n} B_{d-n-1}^{(d-n)}(d-z) B_{n}^{(n)}(z)
$$

in terms of generalised Bernoulli polynomials. For $(25), z_{1}=(d+1) / 2-k, z_{2}=$ $(d+1) / 2+k$.

Fig.2. Dirac effective action, $d=5$

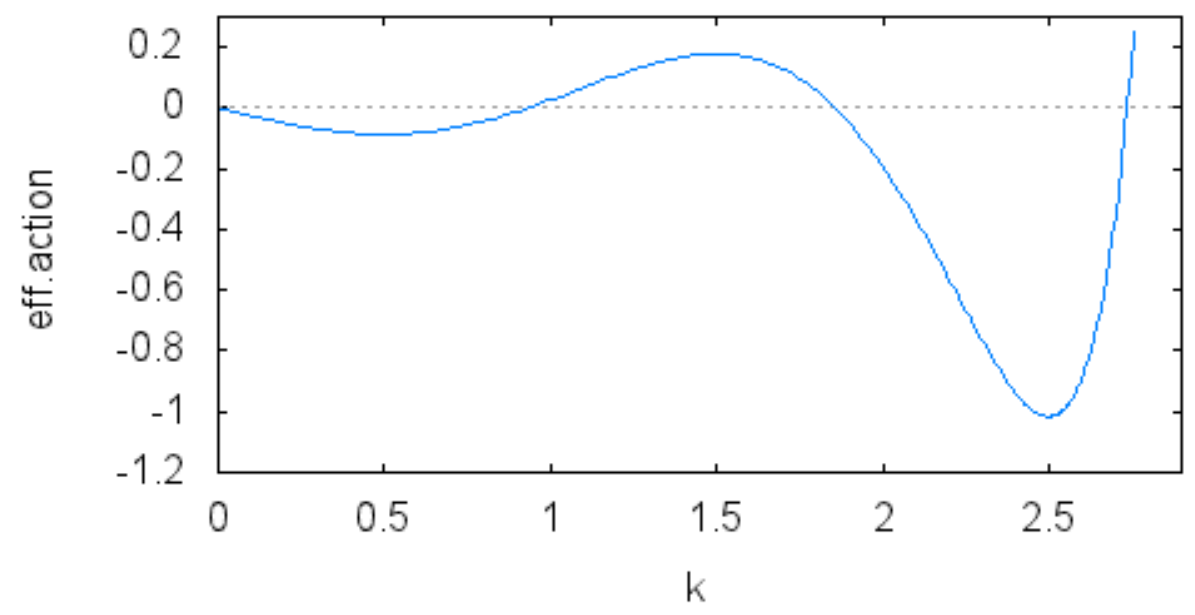

Fig.3. Dirac effective action, $d=7$

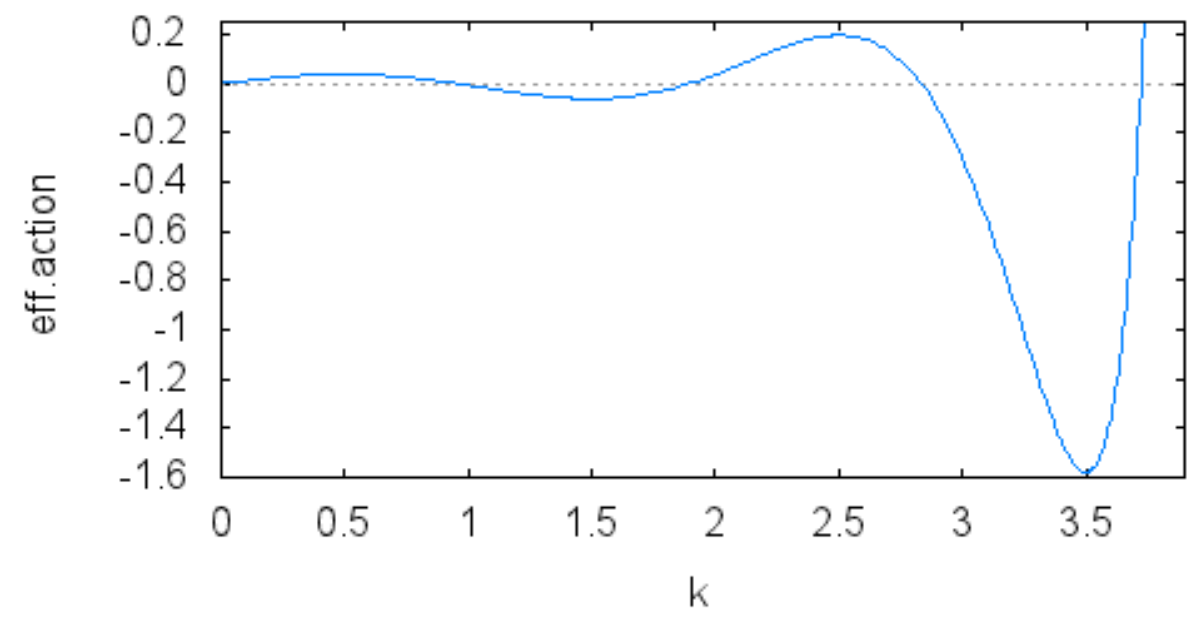

Figures 2 to 4 plot the effective action for several dimensions over the relevant ranges of $k$ and show a series of extrema at half integers. I recall that $k=1 / 2$ 
corresponds to the usual Dirac case. The values agree with those that are available in [6]. Figure 5 is for an even dimension $(d=6)$ the two curves illustrating the effect of leaving out the multiplicative anomaly.

Fig.4. Dirac effective action, $d=3$

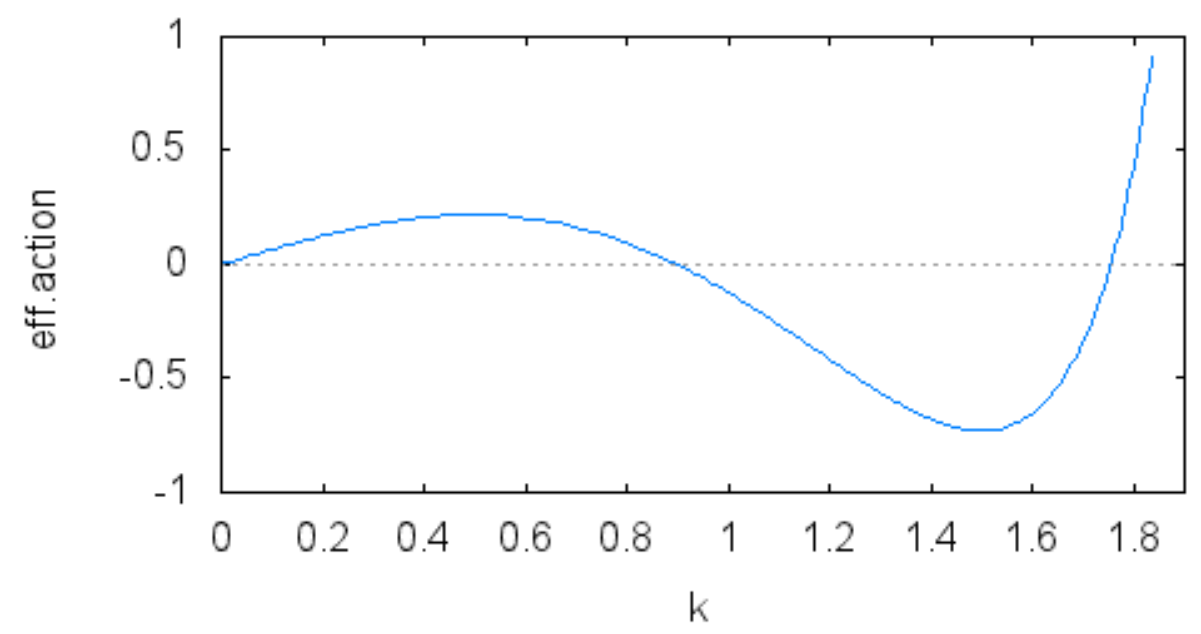

Fig.5. Dirac effective action, $d=6$

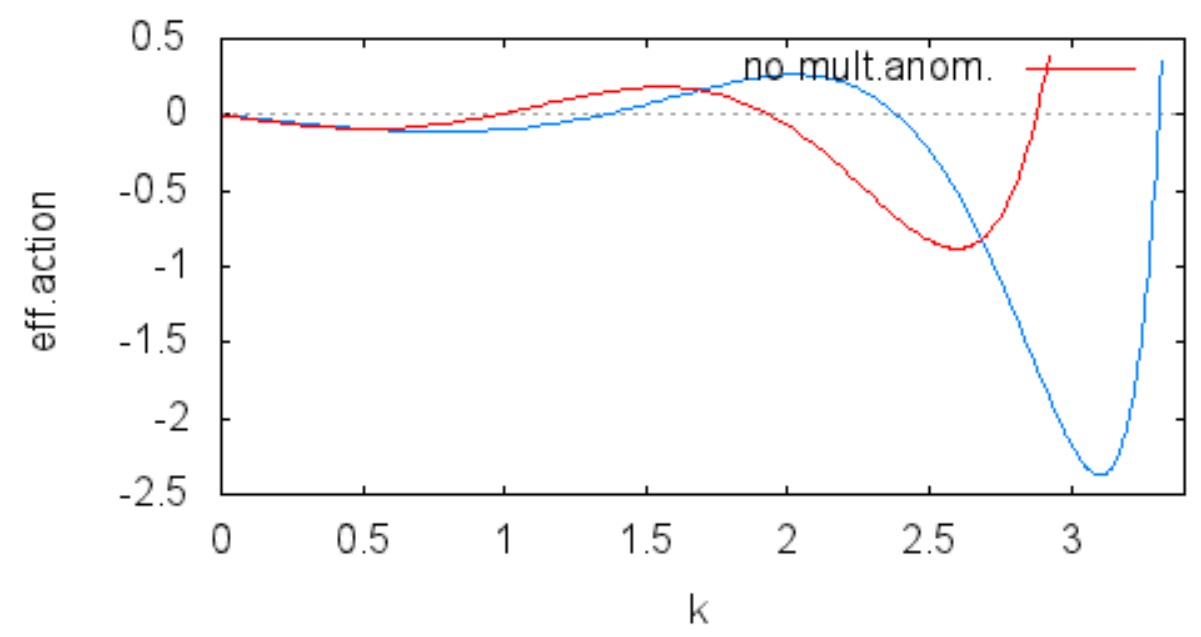

\section{Alternative treatment of determinant for odd dimensions}

In [20] I gave an expression for the scalar determinant on odd spheres in the form of an integral, different from the one corresponding to (28), which was also suitable for numerical calculation. In this section I wish to do the same for the Dirac 
GJMS case. The details are a little different as I deal, first, with the factorisation (8) so that the ordinary Dirac values can be recovered when $l=0$.

From (8),

$$
Z_{d}^{\prime}(0, a, l)=-\log \operatorname{det} C_{l}=-\frac{1}{2} \log \operatorname{det} B^{2}-\sum_{h=1}^{l} \log \operatorname{det}\left(B^{2}-h^{2}\right),
$$

using, again, the absence of a multiplicative anomaly in odd dimensions.

The expression is treated using a Bessel Fourier transform as in [20] where proper references can be found. The detailed algebra is in [20] and so I just give the result I want,

$$
-\log \operatorname{det}\left(B^{2}-h^{2}\right)=\mathcal{S}_{d} \frac{1}{2^{d-1}} \int_{\mathcal{C}} d \tau \frac{\cosh (\omega-a) \tau \cosh h \tau}{\tau \prod_{i=1}^{d} \sinh \left(\omega_{i} \tau / 2\right)}
$$

where the contour, $\mathcal{C}$, runs above all the real axis and below the first singularity of the integrand with a positive imaginary part.

For the total expression, (35), the sum gives the Dirichlet kernel,

$$
-\log \operatorname{det} C_{l}=\mathcal{S}_{d} \frac{1}{2^{d}} \int_{\mathcal{C}} d \tau \frac{\cosh (\omega-a) \tau}{\tau \prod_{i=1}^{d} \sinh \left(\omega_{i} \tau / 2\right)} U_{2 l}(\cosh \tau / 2),
$$

where $U_{2 l}$ is a Chebyshev polynomial. Referring to the eigenvalues (1) $a=d / 2$ and since, for the round sphere, $\omega \equiv \sum_{i} \omega_{i} / 2=d / 2$ we see that the cosh factor gives unity. So, on the $d$-sphere, for spin-half,

$$
F_{l}(d) \equiv-\frac{1}{\mathcal{S}_{d}} \log \operatorname{det} C_{l}(d)=\frac{1}{2^{d}} \int_{\mathcal{C}} d \tau \frac{1}{\tau \sinh ^{d}(\tau / 2)} U_{2 l}(\cosh \tau / 2),
$$

For comparison I give the corresponding spin-0 result,[21],

$\log \operatorname{det} P_{2 k}(d)=-\frac{1}{2^{d-1}} \int_{\mathcal{C}} d \tau \frac{1}{\tau \sinh ^{d}(\tau / 2)} \cosh (\tau / 2) U_{2 k-1}(\cosh \tau / 2)$.

Now, as in the earlier, scalar calculation, I re-expand the Chebyshev polynomial, this time in even powers of $\sinh \tau / 2$,

$$
\begin{aligned}
U_{2 l}(\cosh \tau / 2) & =2 \sum_{i=0}^{l} \frac{(-1)^{i} 2^{2 i}}{(2 i+1) !}(l+1 / 2)^{[2 i+1]} \sinh ^{2 i}(\tau / 2) \\
& \equiv \sum_{i=0}^{l} 2^{2 i} A_{i}(l) \sinh ^{2 i}(\tau / 2)
\end{aligned}
$$


using central factorial notation.

Hence one obtains the decomposition sum rule,

$$
F_{l}(d)=\sum_{i=0}^{l} A_{i}(l) F_{0}(d-2 i)
$$

in terms of the ordinary Dirac values, $\sim F_{0}$, at dimensions $\leq d$ and $\geq d-2 l$. This would be one way to evaluate the Dirac GJMS determinants. Numerically it is less efficient than (36), but has algebraic consequences.

Before discussing these I return, for comparison, to the scalar case, expressed slightly differently to [21]. The expansion required is now,

$$
\begin{aligned}
U_{2 k-1}(\cosh \tau / 2) & =2 \cosh (\tau / 2) \sum_{i=0}^{k-1} \frac{(-1)^{i} 2^{2 i}}{(2 i+1) !} k^{[2 i+2]-1} \sinh ^{2 i}(\tau / 2) \\
& \equiv \cosh (\tau / 2) \sum_{i=0}^{k-1} B_{i}(k) \sinh ^{2 i}(\tau / 2)
\end{aligned}
$$

Substituted into (37), this gives

$$
-\log \operatorname{det} P_{2 k}=\frac{1}{2^{d-1}} \int_{\mathcal{C}} \frac{d \tau}{\tau}\left(\frac{1}{\sinh ^{d}(\tau / 2)}+\frac{1}{\sinh ^{d-2}(\tau / 2)}\right) \sum_{i=0}^{k-1} B_{i}(k) \sinh ^{2 i}(\tau / 2),
$$

which shows that the basic integral required is

$$
\int_{\mathcal{C}} d \tau \frac{1}{\tau \sinh ^{d}(\tau / 2)}=2^{d} F_{0}(d)
$$

from (36) and, therefore, that the scalar GJMS determinant is also expressed as a sum of ordinary Dirac determinants. The lowest order case is

$$
-\log \operatorname{det} P_{2}(d)=2\left(F_{0}(d)+F_{0}(d-2)\right),
$$

where $P_{2}$ is the Yamabe-Penrose conformally invariant Laplace operator. This relation reflects properties of the eigenvalues. It can be inverted to give the spin$1 / 2$ quantity in terms of a sum of scalar ones at varying dimensions,

$$
F_{0}(d)=\frac{1}{2} \sum_{i=1}^{d}(-1)^{i+1} \log \operatorname{det} P_{2}(i)
$$

which is of no numerical interest but has a curiosity value. 
In a similar vein I note that the basic recursion for the Chebyshev polynomials (just trigonometry),

$$
U_{n+1}(x)=2 x U_{n}-U_{n-1}(x),
$$

gives a recursion on the order of the operators,

$$
-\log \operatorname{det} P_{2 k}(d)=F_{k}(d)+F_{k-1}(d)
$$

again somewhat of a curiosity.

As mentioned, the ordinary Dirac values are known but I will discuss them again using the approach of this section.

So far as explicit computation is concerned one way is to transcribe the contour integral into real form by setting $\tau=x+i \pi$ and using the symmetry of the integrand to give

$$
\begin{aligned}
F_{0}(d) & =\frac{(-1)^{(d+1) / 2}}{2^{d-1}} \int_{0}^{\infty} d x \frac{\operatorname{sech}^{d}(\pi x / 2)}{x^{2}+1} \\
& \equiv \frac{(-1)^{(d+1) / 2}}{2^{d-1}} J(d)
\end{aligned}
$$

where $J(d)$ is the same integral that occurs in the scalar case, [20], in accordance with the expression (40).

The integral $J(d)$ was obtained in [20] as a combination of Riemann $\zeta$-functions (actually, Dirichlet $\eta$-functions) and $\log 2$ by a method which involved expanding the sech $^{d}$ in derivatives of sech. The coefficients appeared as, [22], central differentials of zero, $D^{r} O^{[s]}$ (e.g. Steffensen, [23]),

$$
\begin{gathered}
J_{2 m+1}=(-1)^{m} \sum_{n=1}^{m}(-1)^{n} \frac{2^{2(m-n)} D^{2 n+1} 0^{[2 m+1]}}{\pi^{2 n}(2 m) !(2 n+1)} \eta(2 n) \\
+(-1)^{m} D 0^{[2 m+1]} \log 2 .
\end{gathered}
$$

A similar form results from pushing the contour $\mathcal{C}$ in (36) upwards to infinity, and employing residues, in the fashion of Candelas and Weinberg, [24]. A systematic discussion, starting from (41), is given in [21] the coefficients now appearing as higher $D$-Nörlund (Bernoulli) numbers from the power series of $\operatorname{cosech}^{d}$ (see below). The equality of the two expressions is guaranteed by (or is a proof of) an identity between these numbers and the differentials of zero (or, equivalently, the central factorial numbers of the first kind), see [21].

The contour method in [21] effectively employs the decompositions like (40), the quantity denoted there by $f_{2 m+1}$ equalling $J(2 r+1) / \pi$ in $(41)$. 
The expressions agree with those, e.g., in [6] Table 2, derived by a more conventional eigenvalue method.

The same Bessel technique can be applied to the other factorisation of the 'intertwiner', (22). The algebra is very similar to the scalar case, except that now $a=d / 2$ so that, instead of (37), there appears, $(k \in \mathbb{Z})$,

$$
-\log \operatorname{det} \bar{C}_{k}(d)=\frac{\mathcal{S}_{d}}{2^{d-1}} \int_{\mathcal{C}} d \tau \frac{1}{\tau \sinh ^{d}(\tau / 2)} U_{2 k-1}(\cosh \tau / 2) .
$$

As before, this integral can be treated numerically as it stands but again I employ the expansion (39) to give,

$$
\begin{aligned}
-\log \operatorname{det} \bar{C}_{k}(d) & =\frac{\mathcal{S}_{d}}{2^{d-1}} \sum_{i=0}^{k-1} B_{i}(k) \int_{\mathcal{C}} d \tau \frac{\cosh (\tau / 2)}{\tau \sinh ^{d-2 i}(\tau / 2)} \\
& =-\frac{\mathcal{S}_{d}}{2^{d-2}} \sum_{i=0}^{k-1} \frac{B_{i}(k)}{d-2 i-1} \int_{\mathcal{C}} d \tau \frac{1}{\tau^{2} \sinh ^{d-2 i-1}(\tau / 2)}
\end{aligned}
$$

which, out of interest, I evaluate by residues using the standard expansion,

$$
(t \operatorname{cosech} t)^{d}=\sum_{\nu=0}^{\infty} \frac{D_{2 \nu}^{(d)}}{(2 \nu) !} t^{2 \nu},
$$

in terms of $D$-Nörlund numbers. Basic algebra gives the double summation,

$$
\begin{aligned}
& -\frac{\log \operatorname{det} \bar{C}_{k}(d)}{\mathcal{S}_{d}}=\frac{(-1)^{(d-1) / 2}}{2^{d-1}} \sum_{i=0}^{k-1} \frac{(-1)^{i}}{\pi^{d-2 i-1}} B_{i}(k)[ \\
& \left.\sum_{\nu=0}^{(d-3-2 i) / 2} \frac{(-1)^{\nu}}{(2 \nu) !} \frac{d-2 i-1-2 \nu}{d-2 i-1} D_{2 \nu}^{(d-2 i-1)} \pi^{2 \nu} \zeta_{R}(d-2 i-2 \nu)\right],
\end{aligned}
$$

which is a sum of Riemann, not Dirichlet, zetas. Also there is no $\log 2$.

The simplest case is the operator for $k=1$ i.e. $\bar{C}_{1}(d)=\not^{2}-1 / 4$ from (22). For example, the right-hand side of (42) reduces to $\zeta_{R}(3) / 2 \pi^{2}$ for $d=3$ and to $-\zeta_{R}(3) / 24 \pi^{2}-\zeta_{R}(5) / 8 \pi^{4}$ for $d=5$, and so on. The next case is the Paneitz operator when $k=2$, i.e. $\bar{C}_{2}(d)=\left(\not^{2}-1 / 4\right)\left(\nabla^{2}-9 / 4\right)$. For $d=5$ there results $\zeta_{R}(5) / 4 \pi^{4}-5 \zeta_{R}(3) / 12 \pi^{2}$. Numerical quadrature from the real form of (43) gives agreement to at least 14 places. While this is really only a check of the algebra it does reveal that quadrature is more efficient. 


\section{Conclusion and comments}

The conformal anomalies and functional determinants have been derived, and computed, for spin-half GJMS operators on spheres using a direct spectral method directly parallel to the corresponding scalar case. Technically, I have again found that the Barnes $\zeta$-function is very handy allowing one to avoid expanding degeneracies and the resulting Riemann/Hurwitz $\zeta$-functions. However, these reappear via a different route.

The determinants calculated for two factorisations of the general $\Gamma$-function form of the GJMS operator (the intertwiner) were shown to be equal when continued, including the multiplicative anomalies.

The results, which have a holographic aspect, can also be derived formally by a tensor product construction as in [11] but I leave this for another time. I also postpone discussion of the fact that counting the number of negative modes, as $k$ varies, of the GJMS operator on the $d$-sphere gives the degeneracies of $\nabla^{2}$ on the $(d+1)$-sphere.

An alternative approach for odd dimensions yielded the answer for the (log) determinant as a quadrature. Further analytical work turned this into the familiar sum of Dirichlet $\eta$ - functions and $\log 2$. For the other factorisation, just a sum of Riemann zetas appears.

\section{References.}

1. Tseytlin,A.A. On Partition function and Weyl anomaly of conformal higher spin fields ArXiv:1309.0785.

2. Aros,R. and Diaz,D.E. Determinant and Weyl anomaly of Dirac operator: a holographic derivation, ArXiv:1111.1463.

3. Diaz,D.E. and Dorn,H. JHEP 0705 (2007) 46.

4. Diaz,D.E. JHEP 0807 (2008) 103.

5. Allais, A. JHEP 1011 (2010) 040.

6. Klebanov,I.R., Pufu,S.S. and Safdi,B.R. JHEP 1110 (2011) 038.

7. Dowker,J.S. and Pettengill,D.F. J. Phys. A7 (1974) 1527

8. Dowker,J.S. Phys. Rev. D28 (1983) 3013.

9. Chang,P. and Dowker,J.S. Nucl. Phys. B395 (1993) 407.

10. Dowker,J.S. Comm. Math. Phys. 162 (1994) 633.

11. Dowker,J.S. J. Phys. A44 (2011) 115402.

12. Cappelli,A. and D'Appollonio, Phys. Lett. 487B (2000) 87. 
13. Copeland,E. and Toms,D.J. Class. Quant. Grav. 3 (1986) 431.

14. Barnes,E.W. Trans. Camb. Phil. Soc. 19 (1903) 374.

15. Dowker,J.S. Numerical evaluation of spherical GJMS operators for even dimensions ArXiv:1310.0759.

16. Gubser,S.S and Klebanov,I.R. Nucl. Phys. B656 (2003) 23.

17. Graham,C.R. SIGMA 3 (2007) 121.

18. Morpurgo,C. Duke Math. J. 114 (2002) 477.

19. Erdelyi, A., Magnus, W., Oberhettinger, F. and Tricomi, F.G. Higher Transcendental Functions Vol.2 (McGraw-Hill, N.Y. 1953).

20. Dowker,J.S. J. Phys.A:Math.Theor. 46 (2013) 2254.

21. Mansour,T. and Dowker,J.S. Evaluation of spherical GJMS determinants, 2014, Submitted for publication.

22. Dowker,J.S., Central differences, Euler numbers and symbolic methods, ArXiv:1305.0500.

23. Steffensen,J.F. Interpolation, (Williams and Wilkins, Baltimore, 1927).

24. Candelas,P. and Weinberg,S. Nucl. Phys. B237 (1984) 397. 